\title{
Niels Bohr and the Formalism of Quantum Mechanics
}

\author{
Dennis Dieks \\ History and Philosophy of Science \\ Utrecht University \\ d.dieks@uu.nl
}

\begin{abstract}
It has often been remarked that Bohr's writings on the interpretation of quantum mechanics make scant reference to the mathematical formalism of quantum theory; and it has not infrequently been suggested that this is another symptom of the general vagueness, obscurity and perhaps even incoherence of Bohr's ideas. Recent years have seen a reappreciation of Bohr, however. In this article we broadly follow this "rehabilitation program". We offer what we think is a simple and coherent reading of Bohr's statements about the interpretation of quantum mechanics, basing ourselves on primary sources and making use of - and filling lacunas in - recent secondary literature. We argue that Bohr's views on quantum mechanics are more firmly connected to the structure of the quantum formalism than usually acknowledged, even though Bohr's explicit use of this formalism remains on a rather global and qualitative level. In our reading, Bohr's pronouncements on the meaning of quantum mechanics should first of all be seen as responses to concrete physical problems, rather than as expressions of a preconceived philosophical doctrine. In our final section we attempt a more detailed comparison with the formalism and conclude that Bohr's interpretation is not far removed from present-day non-collapse interpretations of quantum mechanics.
\end{abstract}

\section{Introduction}

Bohr's writings on the interpretation of quantum mechanics, from his 1927 Como lecture to his articles from the 1950s, are conspicuous by the absence of detailed discussions of technical aspects of the quantum formalism. In this respect they are very different from the tradition of foundational work that 
has started in the early 1950s, with Bohm's hidden-variables scheme, and has led via Bell's theorem and similar results to present-day work on quantum information theory. The predominantly qualitative character of Bohr's arguments, together with his convoluted and sometimes confusing style, can easily create the impression of a rhetoric without substantial support in quantum mechanics itself, aimed at intimidating the reader (Cushing 1994; Beller 1999). That this impression is superficial and misconceives Bohr's perspective on the interpretation of quantum theory has recently been argued from various sides (Howard 1994, 2004, 2005; Faye 2014; Landsman 2006, 2007; Camilleri and Schlosshauer 2015; Zinkernagel 2015, 2016). The present essay is an attempt to further contribute to this program of rehabilitation, without losing sight of possible weaknesses or open questions in Bohr's interpretation. We shall argue that the non-technical nature of Bohr's writing should not be misconstrued: Bohr's views are more intimately connected to the mathematical structure of quantum mechanics than usually acknowledged and reflect salient features of it. In fact, Bohr's interpretation bears affinity to modern non-collapse interpretations of quantum mechanics.

The idea that Bohr's statements about the meaning of quantum mechanics have a direct link to the structure of the mathematical formalism may at first sight seem implausible. After all, Bohr himself appears to downplay the significance of this formalism: from the Como lecture to his latest writings Bohr emphasizes the merely "symbolic" character of the mathematical apparatus that is used in the new theory. This, together with the notorious fact that classically describable measuring devices and measurement outcomes play a central role in Bohr's interpretation, may suggest that he saw the formalism as possessing only pragmatic value, as a tool for making predictions on the macroscopic level. This would imply that Bohr championed instrumentalism, perhaps even denying the existence of a quantum world at all - a position that has indeed not infrequently been ascribed to Bohr and used as a reductio of his views by some anti-Copenhagenists (Cushing 1994; Beller 1999, cf. Faye 2014).

Recent scholarship has questioned this attribution of instrumentalism to Bohr (Folse 1985; Howard 1994; Camilleri and Schlosshauer 2015; Zinkernagel 2015, 2016) - a line that we shall follow and elaborate. To start with, we need to take a closer look at Bohr's reasons for calling the formalism of quantum mechanics "symbolic". After this, we shall pay attention to the role of classical concepts, which in turn will lead us to complementarity and measurement. We conclude with a discussion of how Bohr's views compare to the mathematical formalism, from a modern point of view. 


\section{The symbolic character of the quantum formal- ism}

Already in the first section of his famous 1927 Como lecture Bohr typifies quantum mechanics as a "symbolic method" (Bohr 1928). Characteristically, to argue his point he does not start from the newly developed Schrödinger wave mechanics or Heisenberg matrix mechanics, but discusses the older, simpler and more qualitative ideas of de Broglie (though it should not go unnoticed that in section 5 of the Como lecture Bohr makes it clear that he considers de Broglie's conceptions as having been embedded and generalized in the new wave mechanics). De Broglie had proposed to associate waves with particles: if $E$ denotes the energy and $p$ the momentum of a particle, de Broglie postulates an associated wave with frequency $\nu$ and wavelength $\lambda$ satisfying the relations $E / \nu=p \lambda=h$ (with $h$ Planck's constant). Bohr now observes that according to these relations the phase velocity of the wave associated with a particle moving at speed $v$ is given by $c^{2} / v$, whereas the group velocity is equal to $v$. He comments: "The circumstance that [the phase velocity] is in general greater than the velocity of light emphasizes the symbolic character of these considerations. At the same time, the possibility of identifying the velocity of the particle with the group-velocity indicates the field of application of space-time pictures in the quantum theory."

The contrast that Bohr notes here gives us a first clue about the criteria he has in mind when he qualifies the formalism as "symbolic". It is clear that a wave propagating with superluminal velocity cannot correspond to something physically real propagating in space - this wave must therefore be symbolic in the uncontroversial sense of being not physical but rather having the status of a mathematical tool coming from Fourier analysis. On the other hand, a packet of such mathematical waves, propagating with group velocity $v$, apparently can be thought of as representing something physically real (taking into account caveats and restrictions to be discussed!). Elaborating on the latter point, Bohr goes on to discuss the spreads (dispersions) in energy and momentum inherent in de Broglie wave packets and concludes that these determine "the highest possible accuracy in the definition of the energy and momentum of the individuals associated with the wave-field". We shall soon return to the meaning of this "accuracy in definition"; but an immediate and obvious conclusion to be drawn is that according to Bohr we are allowed to think of physical "individuals" associated with packets of de Broglie waves. Indeed, the Como Lecture is quite explicit that the wave 
packets correspond to atomic and subatomic entities, like electrons. The notion that the lecture is meant to promulgate an instrumentalist interpretation of quantum theory according to which the whole formalism possesses only mathematical and no physical descriptive content is thus immediately seen to sit uneasily with the textual evidence.

It is true that Bohr immediately follows up his mention of quantum individuals with the warning that the applicability of familiar classical concepts to them is severely limited, in view of the "inaccuracy in definition" just mentioned. Energy and momentum, and also position as characterized by a wave packet, cannot possess sharp values. As Bohr tells us, a corollary of this is that classical mechanics can at most have restricted validity. Only in limiting situations, comparable to the limit in which geometrical optics can replace wave optics, is it to be expected that we can recover a picture in which energy and momentum are well-defined in combination with sharp positions (sharp positions at each instant of time lead to "a well-defined space-time picture" in Bohr's terminology). Arguing from the simple example of a single particle, formally represented by a de Broglie wave packet, Bohr at this point already formulates the thesis that well-defined space-time pictures and "the claims of causality" (i.e. the instantiation of well-defined values of energy and momentum fulfilling conservation laws), are not compatible in the description of quantum individuals. As he says,

the content of the relations (2) [i.e. the qualitative uncertainty relations $\triangle t \triangle E=\triangle q \triangle p=h$ derived from a Fourier analysis of wave packets] may be summarised in the statement that according to the quantum theory a general reciprocal relation exists between the maximum sharpness of definition of the space-time and energy-momentum vectors associated with the individuals. ... At the same time, however, the general character of this relation makes it possible to a certain extent to reconcile the conservation laws with the space-time coordination of observations, the idea of a coincidence of well-defined events in a space-time point being replaced by that of unsharply defined individuals within finite space-time regions (Bohr 1928, sec. 2).

This first discussion of the symbolic character of the quantum formalism at the very start of the Como lecture is illuminating and already clarifies several issues. Importantly, that the formalism is symbolic is apparently not meant to imply that the formalism remains completely silent about physical entities on the micro level or even denies their existence. Quite the opposite, Bohr explicitly tells us that the theory is about physical individuals 
like electrons and photons. But, second and certainly not less important, these "things" should not be thought of as objects describable with values of physical quantities like energy, momentum and position in the way we would expect on the basis of classical mechanics. In classical mechanics particles necessarily possess well-defined positions, momenta and energies at all instants - but for quantum individuals the attribution of both sharp spacetime positions and sharp energy/momentum values is inconsistent with the formalism (namely the mathematical properties of wave packets, which can be carried over to the Schrödinger theory), says Bohr. These two groups of concepts (dynamical versus space-time concepts) are incompatible (in fact, "complementary", as to be discussed more extensively in a moment) and their applicability is reciprocally restricted by the uncertainty relations. At the same time, it is possible to think of individuals with "unsharply defined momentum/energy and space-time locations"; and in situations in which Planck's constant $h$ can be considered insignificantly small this gives us an approximation to the classical picture. However, in general the incompatibility of $p$ and $q$ stands in the way of a vizualizable picture of the kind we are used to in classical mechanics and in everyday experience. ${ }^{1}$

So it turns out that the "symbolic character" of the formalism refers first and foremost to the notion that familiar looking mathematical quantities like $p$ and $q$ do not stand for the well-defined physical properties that we would expect them to represent - the relation between the mathematical formalism and the quantum world must consequently be more indirect than in classical mechanics. It does not follow from this that the quantum formalism deals only with macroscopic measurement outcomes and has nothing to say about features of the quantum world. Quite the opposite, Bohr clearly takes the symbolism to yield information about what we can say about quantum individuals like atoms, photons and electrons. In fact, it is the formalism that shows us that such "particles" cannot be pictorially represented in the way of classical physics.

This reading of the meaning of "symbolic" is supported by the remainder of the Como lecture. Bohr briefly discusses matrix mechanics, and observes that $p$ and $q$ in this formulation of quantum mechanics are not numbers, but rather non-commuting matrices obeying the canonical commutation re-

\footnotetext{
${ }^{1}$ At the end of the Como lecture Bohr puts this in the perspective of science leading us ever further away from everyday intuitions: although we make contact with the world through "ordinary perception", to which each and every word in our language is tailored, we have to adapt our theoretical interpretations of these perceptions to "our gradually deepening knowledge of the laws of Nature". Bohr adds that this same point should already have become evident from Einstein's theory of relativity.
} 
lation. As he says, "this exchange relation [i.e. the commutation relation] expresses strikingly the symbolic character of the matrix formulation of the quantum theory." The point is that matrices are operators and not numerical quantities, and therefore cannot directly represent values of physical quantities.

Bohr then turns to wave mechanics, of which Schrödinger had hoped that it would restore visualizability to atomic physics: Schrödinger aimed to relate his $\Psi$ to physical waves existing in three-dimensional space. Bohr rejects this possibility of a vizualizable depiction and asserts that "wave mechanics just as the matrix theory ... represents a symbolic transcription of the problem of motion of classical mechanics adapted to the requirements of quantum theory" (Bohr 1928, sec. 5); and "in the wave equation, time and space as well as energy and momentum are utilised in a purely formal way."

Bohr explains that he has three grounds for the latter claim. First, the Schrödinger theory represents momentum by a differential operator $p=$ $i \hbar \partial / \partial q$, in which the imaginary quantity $i$ occurs. Second, "there can be no question of an immediate connexion with our ordinary conceptions because the 'geometrical' problem represented by the wave equation is associated with the so-called co-ordinate space, the number of dimensions of which is equal to the number of degrees of freedom of the system, and hence in general greater than the number of dimensions of the ordinary space." Third, Bohr notes that "Schrödinger's formulation of the interaction problem, just as the formulation offered by matrix theory, involves a neglect of the finite velocity of propagation of the forces claimed by relativity theory."

This third reason is rather surprising, as it belongs to another category of objections than the other two. The non-relativistic character of 1927 quantum mechanics certainly signifies that this version of the theory cannot be completely correct - it will need a relativistic generalization. But this in itself is not relevant for visualizability or for whether the mathematical quantities in the formalism could be potential descriptors of physical reality; classical mechanics has also been superseded by relativity, but this does not make the formalism of classical mechanics automatically "symbolic" in a philosophically interesting sense. One can of course say that the basic incorrectness of non-relativistic quantum mechanics implies that the world is different from what a literal reading of the formalism would suggest. Still, the other two arguments are conceptually different, and more interesting, because they bear on the representational capacity in principle of the mathematical symbols to capture elements of physical reality, and not on the predictive correctness of the theory. 
That the three arguments are here lumped together as if they were of the same sort appears to betray a certain lack of philosophical finesse on Bohr's part, or a lack of interest in matters of this kind - it is indicative of a physicist's rather than a philosopher's attitude. In the practice of physics research it is quite common not to be too "nitpicking" about small conceptual differences of this sort. And then, of course, the Como lecture was meant for a physics audience - its text is full of detailed examples from contemporary experimental practice and theoretical explanations offered by colleague physicists. This accords with the general point that Bohr is here concerned, as a physicist, with questions about the validity, applicability, consistency and content of the new physical theory. It would accordingly be a distortion to see Bohr's views as basically stemming from an a priori philosophical background: assumptions of physical common sense plus the abstract reasoning typical of theoretical physics suffice to understand his reasoning in the Como lecture and later writings.

The argument that imaginary quantities occur in the theory and that for this reason the theory must be considered symbolic appears as rather unsophisticated from a philosophical viewpoint as well, since imaginary numbers can be used very well to represent physically real quantities. Of course, one should not come under the spell of the everyday language meaning of "imaginary" and confuse it with the mathematical significance of the term; one should not suppose that $i=\sqrt{-1}$ cannot represent anything physically existing because of this connotation. Apart from the trivial point that it is a matter of conventional choice of scale whether measurement results are expressed in real or imaginary numbers, there is the practical example of physically real vector quantities that can be handled very conveniently with complex numbers, as is standard practice in classical electrodynamics. ${ }^{2}$

Nevertheless, in the case before us sense can perhaps be made of Bohr's conclusion. Indeed, suppose we have defined a scale for physical position $q$ in the usual way, i.e. by means of real numbers. Then the relation $p=i \hbar \partial / \partial q$ seems to indicate that real values for $q$ cannot go together with real values for $p$, assuming that the function that is being differentiated is real-valued. This would entail that $p$ and $q$ cannot be jointly defined and measured on the same type of number scale - something very strange from the viewpoint

\footnotetext{
${ }^{2}$ The remark that the occurrence of the imaginary number $i$ signals the symbolic nature of a formalism appears without further explanation passim in Bohr's publications. It seems therefore not completely impossible that Bohr did fall prey to a confusion between the technical mathematical and the intuitive meaning of "imaginary" - if so, this would reinforce the comment that not too much subtle philosophizing should be imputed to Bohr.
} 
of classical physics and impossible to visualize. If on the other hand the wave function that is differentiated has an imaginary value itself (as is actually common in quantum mechanics), this wave is apparently of a completely different dimension than the physical quantity $q$, which is also impossible to incorporate into a classical picture. So the occurrence of $i=\sqrt{-1}$ may in the present context be associated with the incompatibility of physical quantities. The symbols representing these quantities in the formalism must therefore be symbolical in the same sense as before, namely as defying vizualizability and literal interpretation.

Bohr himself tells us that his second argument, about the dimensionality of configuration space, is the most important one: "above all there can be no question of an immediate connexion with our ordinary conceptions because...the wave equation is associated with the so-called co-ordinate space." Indeed, this argument seems clear and illustrative of what Bohr is aiming at. The Schrödinger wave in the case of a many-particle system is not a physical wave in three-dimensional space (which would be an "ordinary conception") but "lives" in a high-dimensional mathematical space, so it is "symbolical" in the sense we discussed.

This argument does not entail that the wave function cannot contain information about the micro world: the description of a many-particles system (like a gas) by means of one point in phase space, in classical statistical mechanics, also uses many more than three dimensions, but is still in one-to-one correspondence with the ordinary description of several particles in threespace. So we should not mistake this argument for the symbolic character of the wave function for an argument in favor of instrumentalism.

In fact, the analogy with classical statistical mechanics shows that Bohr's argument is more complicated than it first appears. The argument only acquires cogency when we take into account that the situation in quantum mechanics is fundamentally different from the one in classical statistical mechanics: a quantum many-particles wave in configuration space cannot in general be decomposed into many single-particle three-dimensional waves, whereas a classical phase point in a high-dimensional phase space can be understood in terms of ordinary three-dimensional descriptions of individual particles. This difference is due to the possibility of superposing quantum waves in configuration space: the sum of any two waves is again a bona fide Schrödinger wave. Even if the two original waves were decomposable as a product of one-particle waves, the superposed wave would not be. This relates directly to the typical quantum feature of entanglement and the corresponding holistic character of the quantum description of many-body systems. Bohr does not mention the point in this form (entanglement), but 
we have to assume that the superposition principle was in the back of his mind when he argued about the symbolic character of the description in configuration space.

Essentially the same arguments as used in the Como lecture can be found in Bohr's later work (although, perhaps significantly, the argument about the non-relativistic character of the theory disappears). In his famous "Discussion with Einstein on epistemological problems in atomic physics" Bohr explicitly connects the symbolic character of the quantum formalism to its non-vizualizability when he speaks of "the use of not directly visualizable symbolism" (Bohr 1949, 238), and he extensively comments on the difficulty of thinking of quantum objects in terms of conventional physical attributes. ${ }^{3}$ In his brief 1948 Dialectica article in which he summarizes his position we again read: "These symbols [viz. $p$ and $q$ ] themselves, as is indicated already by the use of imaginary numbers, are not susceptible to pictorial interpretation" (Bohr 1948, 314). In the same article Bohr freely speaks about atomic objects, electrons and photons; while emphasizing again the novel quantum problem of describing them in terms of the usual physical quantities.

We may conclude that Bohr's emphasis on the symbolic nature of the quantum formalism is intended to warn us against a literal classical interpretation with respect to the meaning of mathematical quantities like $p$ and $q$. The quantum world should accordingly not be thought of as directly mirrored by the mathematical formalism: micro properties cannot be read off from the mathematical symbols in the manner they can in classical mechanics. Moreover, Bohr argues that the problems that we encounter when we try to construct such a classical picture, while staying in conformity with quantum mechanics, are not of a pragmatic nature: they constitute problems of principle. The very structure of the quantum formalism precludes its classical interpretation.

The arguments that Bohr adduces for non-vizualizability and the symbolic nature of the formalism therefore by the same token show that the structure of the quantum formalism provides us with information about features of the quantum world. For example, we saw that the spreads of wave packets according to Bohr indicate to what extent a space-time picture and/or a causal description are applicable, and therefore to what extent such descriptions are able to latch on to the physical world. Directly related to this, the canonical commutation relations in the mathematical formalism

\footnotetext{
${ }^{3}$ Thereby recognizing the existence of quantum objects, even if not characterizable in a detached and non-contextual way; note also that Bohr talks about "not directly visualizable formalism" (emphasis added), leaving it open that indirect ways of endowing the symbols with physical content may be possible.
} 
tell us that sharp values of position and momentum cannot co-exist, and so inform us about a characteristic feature of the quantum world.

\section{The indispensability of classical concepts}

The symbolic character of the mathematical formalism of quantum mechanics in the sense just explained implies that we cannot rely on the usual physical interpretation of mathematical symbols like $p$ and $q$. This raises the question of how we can interpret the formalism at all. The dilemma is that to endow the symbols with physical meaning we seem to need knowledge about what the quantum world is like, what kind of things are "out there", so that we can make the formalism correspond to these things. But on the other hand it is quantum mechanics itself that has to provide us with information about the structure of the quantum world. However, the theory can only do so after it has made contact with physical reality, when it is an interpreted physical theory: a purely mathematical scheme cannot tell us anything about the physical world.

A deceptively similar problem played a major role in early twentieth century logical empiricism. The logical empiricists attempted to make it clear how purely mathematical calculi could be interpreted empirically, without the intrusion of metaphysical elements. They proposed to solve this problem via the postulation of "correspondence rules" (Carnap) or "coordinative definitions" (Reichenbach); the idea simply being that we need an already empirically interpreted and stable vocabulary through which symbols in the as yet uninterpreted theory can be infused with physical meaning. Given that it is essential for this logical empiricist enterprise to stay as close as possible to theoretically uninfected experience, it is an obvious choice to take an observation language with a minimum of theoretical baggage for the already interpreted and stable language. The interpretational basis should thus be close to our pre-theoretical everyday language and should enable us to unambiguously refer to observable things and the results of experiments (as in Carnap's "Ding-Sprache"). The various ways in which this basic idea was elaborated by the logical empiricists, and the many ensuing debates (verificationism, cognitive significance, instrumentalism versus realism, etc.), are well known and need not detain us here.

The short description of the basic idea just given already suffices to make it understandable why the logical empiricists expected Bohr to be a close philosophical friend. ${ }^{4}$ But although part of Bohr's ideas about the

\footnotetext{
${ }^{4}$ In 1936 the 2nd International Congress for the Unity of Science took place in Copen-
} 
interpretation of the quantum formalism can be seen as a physicist's version of the correspondence rule programme, there are also important differences. Bohr's approach was not motivated by a strict empiricism or by an anti-metaphysical attitude, but by the problems - reviewed in the previous section - that arise when we wish to give the usual classical interpretation to symbols like $p$ and $q$.

Nevertheless, the task Bohr had to face bears a striking resemblance to the problem of how to give empirical content to an uninterpreted formalism, as we are also here dealing with a formalism that needs empirical interpretation. Moreover, the sought-for interpretation should obviously make it possible to adequately describe what is happening in the laboratory. But in this description of the laboratory world essential differences start to appear between Bohr and the logical empiricists. Bohr does not strive for a purified theory-less observation language (and even less for a reliance on sense data), but simply accepts that the interpreted new theory should be a generalization of classical physics. The anchor point for this generalization are the descriptions we already use in experimental practice: in the laboratory we speak about macroscopic objects and measurement outcomes, but also about many not directly observable things, like atomic particles, electric and magnetic fields, electric currents, and so forth. Bohr does not at all protest against this and does not attempt to suppress or reconstruct this language. However, as we have seen, he does warn against the supposition that all aspects of this "classical language" possess unrestricted validity, and in particular against the uncritical joint attribution of traditional physical attributes when we refer to micro entities. On the other hand, that the assignment of classical attributes works eminently well in the case of macroscopic objects is an unavoidable fact of laboratory life and more than two centuries of experience with classical physics. Quantum theory must certainly be able to reproduce this actual state of affairs. This situation suggests a way for linking the quantum formalism to experience, namely via our already existing classical description of the macro world. As Bohr puts it in an often-quoted passage in his contribution to the 1949 Einstein volume:

It is decisive to recognize that, however far the phenomena transcend the scope of classical physical explanation, the account of

hagen at Bohr's honorary residence at Carlsberg (Faye 2008). Neurath, Hempel, Popper, Philipp Frank and other logical empiricist luminaries were in attendance, whereas Reichenbach, Schlick, and Carnap intended to be present but were finally unable to come. Bohr later complained, however, that the philosophers had not understood his ideas about complementarity. 
all evidence must be expressed in classical terms. The argument is simply that by the word "experiment" we refer to a situation where we can tell others what we have done and what we have learned and that, therefore, the account of the experimental arrangement and of the results of the observations must be expressed in unambiguous language with suitable application of the terminology of classical physics (Bohr 1949, 209).

This passage has been deemed mysterious and has given rise to speculations about why Bohr thought that classical concepts were essential. Thus, it has become one of the sources of the belief that Bohr was an instrumentalist or a strict empiricist. It has also been interpreted as evidence that Bohr was influenced by Kantianism and saw classicality as an a priori precondition of the possibility of empirical knowledge; or that he anticipated the more modern philosophical idea that we are "suspended in language", in fact classical language. ${ }^{5}$

But motivations originating in - or strongly influenced by - philosophical doctrines need not be assumed here. As is clear from the wording of the just quoted passage, Bohr himself thought of his claim as simple and obvious. This is in agreement with our view that Bohr took the classicality of experimental practice (in the sense that classical concepts are adequate for the description of results and are in fact the only ones we possess to describe them) as an undisputed and familiar fact. This classical description is basically just the description in terms of everyday language, generalized by the addition of physics terminology, and it is the one we de facto use to describe our environment. Bohr apparently found this observation so selfevident, almost tautological, that he sometimes called the applicability of classical concepts to experimental practice a "logical demand"; because, as he says, "the word 'experiment' can in essence only be used in referring to a situation where we can tell others what we have done and what we have learned" (Bohr 1948, 313).

This simple reading of Bohr's doctrine of classical concepts is confirmed, as we shall see, by the way Bohr further elaborated on the role of these concepts in his interpretation of quantum mechanics, and also by evidence coming from explanations that Bohr gave in private conversations. For example, in discussions with Heisenberg in 1927 Bohr already stated: "in spite of your uncertainty principle you have got to use words like "position' and 'velocity' just because you haven't got anything else" (Camilleri

\footnotetext{
${ }^{5}$ Faye (2014) and Howard (1994) present a list of such "philosophical interpretations" of Bohr.
} 
and Schlosshauer 2015); and von Weizsäcker tells the anecdote that when the physicist Edward Teller was expressing doubts about the importance of classical concepts, at an afternoon tea at Bohr's Institute, Bohr briefly fell asleep and awakening merely replied: "Oh, I understand. You might as well say that we are not sitting here, drinking tea, but that we are just dreaming all this" (Drieschner 2014, 78).

The use of classical concepts does not involve a wholesale acceptance of classical physics. As Bohr says in the above quotation, it is the evidence provided by macroscopic measuring devices (pointer readings, light flashes, sounds, etc.) that is to be expressed in classical terms, "however far the phenomena transcend the scope of classical physical explanation". In other words, although we need kinematical concepts like $p$ and $q$ on the macroscopic level, we have to expect that the classical dynamical laws will fail to give us adequate explanations of the values that we actually find for these quantities.

Specifically, we know that macroscopic objects can be ascribed a position and a momentum; and Bohr does not hesitate to relate these to the $q$ and $p$ occurring in the quantum formalism when it is applied to macroscopic objects. We here again discern the significant difference between Bohr's concerns, which are primarily physical, and the philosophical concerns of the logical empiricists. Bohr is not interested in formal rules that determine the empirical content of terms like "position" and "momentum", and is not engaged in a conceptual purification programme à la Mach. He accepts the descriptive vocabulary of classical physics as soon as this becomes possible without contradictions, i.e. on the macroscopic level, and uses this as his cue to achieve a generalization of classical physics that applies to atomic objects as well. As we shall see in the next section, this generalization will be realized not by formal definitions of microscopic attributes in terms of macroscopic measuring results (as might be expected on a logical empiricist analysis), but by partly taking over the "metaphysics" of classical physics while complicating it by adding a relational aspect, namely a dependence on the experimental context (the famous doctrine of complementarity). The very first two sentences of section 1 of the Como lecture already announce this: "The quantum theory is characterised by the acknowledgment of a fundamental limitation in the classical physical ideas when applied to atomic phenomena. The situation thus created is of a peculiar nature, since our interpretation of the experimental material rests essentially upon the classical concepts" (Bohr 1928, 580).

However, Bohr's strategy seems to be immediately menaced by the objection that his own arguments for the symbolic nature of the quantum 
formalism forbid an identification of the quantum symbols $p$ and $q$ with the classical concepts of momentum and position, even in the macroscopic realm. At least, this problem arises if we consider quantum mechanics to be a universal theory, applicable both at the micro and macro level. Now, it has often been claimed in the older literature, and is sometimes still said today, that Bohr assumed that the world consists of two ontologically different parts, on two sides of a dividing line, a "cut": a quantum world on the microscopic side of the cut, and a classical world, governed by classical laws, on the macro side.

But the idea that Bohr denied the universal validity of quantum mechanics is mistaken. Indeed, from his earliest writings on Bohr argues from the assumption that quantum theory is universal, in the sense of applicable to both micro and macro systems (Howard 1994; Landsman 2006, 2007; Camilleri and Schlosshauer 2015). ${ }^{6}$ In the Como lecture the universality of quantum theory is not an explicitly addressed theme, but is implicitly assumed throughout the text. For example, already in the first section of the lecture Bohr mentions that observations of atomic systems consist in physical interactions with "the agencies of observation", and that these interactions are to be treated quantum mechanically and, importantly, symmetrically so that "an independent reality in the ordinary physical sense [i.e. the classical sense in which each system has its own independent state] can neither be ascribed to the phenomena nor to the agencies of observation" (Bohr 1928, 580). The lack of independence to which Bohr refers here is a typical quantum feature that we shall discuss further in the next section; the important point for our present purposes is that this quantum feature is ascribed to both parties in a measurement, to the micro system and the measuring device ${ }^{7}$.

In his 1935 reply to Einstein, Podolsky and Rosen, Bohr is more explicit in his discussion of macroscopic devices, and employs quantum mechanics to deal with a macroscopic diaphragm in the experimental set-up that he proposes as an analogue to the EPR experiment. Interestingly, he mentions here that it may seem a problem that this implies using quantum mechanics

\footnotetext{
${ }^{6}$ Zinkernagel $(2015,2016)$ has recently defended a more sophisticated version of a Bohrian ontological "cut", according to which it is true that each physical system can be described quantum mechanically, but not all systems can be treated quantum mechanically at the same time. We shall come back to this "anti-quantum-fundamentalism" shortly.

${ }^{7}$ Moreover, Bohr tells us in the same passage that it is arbitrary how we divide the total system into a measured and a measuring part. This would make no sense at all if there were an ontological "cut" between a quantum and a classical realm.
} 
for "some process to which the ideas of classical mechanics can be applied." But, he says, "if all spatial dimensions and time intervals are taken sufficiently large, this involves clearly no limitation as regards the accurate control of momentum", and "a purely classical account of the measuring apparatus ... implies the necessity of allowing a latitude corresponding to the quantum-mechanical uncertainty relations in our description of its behavior" (Bohr 1935, 698). Bohr implies here that the quantum description remains ontologically primary, even when we use (as we must) classical concepts to describe macroscopic objects. The classical description is approximate in the sense that in principle we should accept that there are "latitudes" in the classical quantities (like $p$ and $q$ ), in accordance with the uncertainty relations. However, these latitudes will normally play no role in our dealings with the macroscopic world because they are enormously small ("conditioned by $h "$ ") compared to the usual values of macroscopic quantities, and they will therefore not stand in the way of a classical description. However, they certainly exist and should be taken into account explicitly (Bohr 1935, 698 second column) when we discuss the consistency of the quantum mechanical scheme - implicitly they are always there, even when we use only classical terms and do not apply the quantum formalism.

Bohr elaborates this point in his Dialectica article and in his contribution to the Einstein volume (Bohr 1948, 1949). In the latter, in his famous analysis of experiments in which a particle goes through a hole (or two holes!) in a diaphragm - prompted by Einstein's arguments at the 1927 Solvay conference - Bohr comments on the possibility of controlling the momentum transfer between the particle and the macroscopic diaphragm:

Here, it must be taken into consideration that the position and the motion of the diaphragm and the shutter have so far been assumed to be accurately co-ordinated with the space-time reference frame. This assumption implies, in the description of the state of these bodies, an essential latitude as to their momentum and energy which need not, of course, noticeably affect the velocities, if the diaphragm and the shutter are sufficiently heavy (Bohr 1949, 215).

The essential point to note here is that Bohr mentions the quantum and classical descriptions of the diaphragm and shutter as jointly applicable. Moreover, the classical description, in terms of a well-defined velocity, is clearly conceived of as approximate (the latitude required by quantum theory will not noticeably affect the definite value of the velocity if the bodies 
are macroscopic in the sense of sufficiently heavy). The same sort of remarks can be found throughout Bohr's analyses of Einstein's thought experiments.

It is true that Bohr also says that we should distinguish "between the objects under investigation and the measuring instruments which serve to define, in classical terms, the conditions under which the phenomena appear" (Bohr 1949, 221-222). But he follows this up with the declaration that

all unambiguous use of space-time concepts in the description of atomic phenomena is confined to the recording of observations which refer to marks on a photographic plate or to similar practically irreversible amplification effects like the building of a water drop around an ion in a cloud-chamber. Although, of course, the existence of the quantum of action is ultimately responsible for the properties of the materials of which the measuring instruments are built and on which the functioning of the recording devices depends, this circumstance is not relevant for the problems of the adequacy and completeness of the quantum-mechanical description in its aspects here discussed.

This shows that Bohr considered it a matter of course that from an ontological point of view macroscopic objects are basically quantum mechanical. He also identifies, in the just-quoted passage, what he sees as the conditions that have to be fulfilled, from the viewpoint of the quantum description, to make classical concepts applicable: effects must be amplified to the macroscopic level and must become practically irreversible. The conclusion must be that for Bohr the necessity of using classical concepts has a purely epistemic status: it has to do with our access to the world, by means of macroscopic devices that are described by common language (extended by classical physics). It certainly does not correspond to any ontological dividing line between quantum and classical.

This conclusion is decisively supported by Bohr's Dialectica paper, in which he summarizes the situation with comparatively great clarity. Bohr explains:

Incidentally, it may be remarked that the construction and the functioning of all apparatus like diaphragms and shutters, serving to define geometry and timing of the experimental arrangements, or photographic plates used for recording the localization of atomic objects, will depend on properties of materials which are themselves essentially determined by the quantum of action. Still, this circumstance is irrelevant for the study of 
simple atomic phenomena where, in the specification of the experimental conditions, we may to a very high degree of approximation disregard the molecular constitution of the measuring instruments. If only the instruments are sufficiently heavy compared with the atomic objects under investigation, we can in particular neglect the requirements of relation (3) [i.e., the uncertainty relation] as regards the control of the localization in space and time of the single pieces of apparatus relative to each other (Bohr 1948, 315-316).

Once again, it should be noted that Bohr here speaks about approximations, and neglecting the quantum character of the macroscopic devices. Obviously, one can only neglect something if it actually exists. It follows that according to Bohr the classical descriptions of devices must in principle be applicable at the same time as their quantum descriptions.

A difference between the two descriptions is that our immediate epistemic access is restricted to the classical one, and that we therefore need this classical description to give physical content to the quantum formalism at all. When we thus use classical language to endow the quantum formulas with physical content, it turns out that we cannot transfer the usual classical pictures to the micro world: even when interpreted with the help of classical concepts, the formalism of quantum mechanics remains non-visualizable in the general case of latitudes in position and momentum that cannot be neglected. This is the background of complementarity, to be discussed in the next section.

We therefore side with Landsman $(2006,2007)$, who briefly remarks that the Bohrian privileged status of classical concepts should be considered as merely epistemic; and also with Camilleri and Schlosshauer (2015) who argue that there is no conflict between Bohr's doctrine of classical concepts and the present-day "decoherence research programme" according to which classical descriptions emerge as very accurate approximations when quantum mechanics is applied to open systems (like the objects of everyday experience).

Our conclusion also bears affinity to what Howard (1994) proposes, but there are differences of nuance. Howard argues that Bohr demanded a classical description only of a part of macroscopic measuring devices, and that a quantum description would be possible for the remaining properties; and, second, that the use of classical concepts can be understood from a more fundamental requirement of "objectivity". The first thesis suggests (perhaps unintentionally) that the classical part of a device cannot be given a 
quantum description at the same time; this would be false, as we think to have demonstrated. It $i s$ true, of course, that to give a quantum description of a device we need the physically interpreted formalism, and therefore some other part of the world to which we apply classical concepts unrestrictedly.

Howard's second thesis, about an underlying philosophical requirement ("objectivity") that should justify the privileged status of classical concepts, in our opinion imputes too much in the way of philosophical motivation and approach to Bohr. In our portrayal, Bohr should first of all be seen as a physicist, motivated by concrete physical problems of his days. That we need classical language is from this viewpoint a simple bare fact, given the actual physical situation we find ourselves in; it is not something in need of philosophical justification. According to Bohr the strangeness of the situation, and what has to be explained, is not that we can apply classical concepts, but rather that classical descriptions cannot be applied across the board. Quantum features of the world manifest themselves in the results of experiments in our laboratories and these results can evidently be described in classical language. So how can we understand that this basic classicality does not extend to the micro world, as demonstrated by wave particle duality (in the Compton effect, for example) and similar phenomena? The task Bohr saw before him was to incorporate this unmistakable micro non-classicality into one consistent whole together with the equally undeniable classicality of our macro descriptions.

According to this analysis Bohr viewed the world as fundamentally quantum mechanical: we live in a quantum world. This contradicts a recent construal of Bohr's ideas by Zinkernagel, who argues that Bohr was not a "quantum fundamentalist". According to Zinkernagel (2015, 2016), Bohr held that although all systems can be treated quantum mechanically, they cannot all be treated this way at the same time.

This in itself is not in conflict with what we said above about the epistemic necessity of describing some systems classically in order to have a pragmatic starting point for the treatment of other systems. But Zinkernagel rejects this epistemic interpretation, and puts an ontological spin on Bohr's statements. He objects to the idea that Bohr's requirement of a classical description of measuring devices is a pragmatic or epistemological demand and also rejects the view that according to Bohr the device is really a quantum mechanical system. For example, he writes:

A way to understand Bohr's requirement is that we need a reference frame to make sense of, say, the position of an electron (in order to establish with respect to what an electron has a po- 
sition). And, by definition, a reference frame has a well-defined position and state of motion (momentum). Thus the reference frame is not subject to any Heisenberg uncertainty, and it is in this sense (and in this context) classical. This does not exclude that any given reference system could itself be treated quantum mechanically, but we would then need another - classically described - reference system e.g. to ascribe position (or uncertainty in position) to the former. I think Bohr's view can be summarized in the claim that any system may in principle be seen as and treated quantum mechanically, but not all systems can be seen as and treated in this way simultaneously. This reading of Bohr's viewpoint might be called restricted quantum universalism (Zinkernagel 2015, 430).

But as we have seen in our earlier quotations of Bohr, Bohr does not maintain that a classical system is not subject to Heisenberg uncertainty; quite the opposite, Bohr claims that it is essential for consistency that this uncertainty exists in principle even in macroscopic objects, in the form of very small latitudes, although it is true that we should necessarily discard these latitudes in a classical description of the object (in which they make no sense). That we need another, classically described, system as soon as we wish to treat a macroscopic object quantum mechanically is true, but as we have seen this can be given a natural epistemological reading. Zinkernagel's ontological interpretation is therefore not well founded in what Bohr writes. What is more, the precise ontological content of his proposed "restricted quantum universalism" is obscure. It is completely intelligible that descriptions of physical systems can be context dependent; this is an epistemological point of whose validity we can easily become convinced even without any appeal to quantum mechanics. But what can it mean to say that the ontological status of an object is context dependent; that in one context it is classical, and in another context it is quantum mechanical, even though these two "modes of being" exclude each other? A systematic elucidation and elaboration of this idea would require a drastic change in basic ideas about ontology and it seems implausible to impute such intentions to Bohr, who hardly makes any explicit reference to ontology at all (whereas epistemological claims abound in his work). 


\section{Measurement and complementarity}

From the very start of quantum theory it has been a leitmotiv that interactions between quantum systems have to be treated in a way that is different from what we are used to in classical physics - quantum theory can be said to have begun with the suggestion that interactions can only take place in the form of exchanges of finite portions of energy and momentum. This basic idea can be traced from Planck's derivation of the black body radiation law and Einstein's 1905 light quantum hypothesis, via Bohr's "old quantum theory" of 1913, to the research in which Bohr was engaged in the 1920s. This discreteness of interactions developed in Bohr's thought from a simple discontinuity in energy exchanges between well-defined physical systems to a fundamentally unanalyzable aspect of interactions and then to the notion that interacting quantum systems are so intertwined with each other that they form a whole that cannot be thought of as consisting of subsystems with their own states. As Bohr puts it in his 1948 concise position statement in Dialectica: "a wholly new situation in physical science was created through the discovery of the universal quantum of action, which revealed an elementary feature of 'individuality' of atomic processes far beyond the old doctrine of the limited divisibility of matter originally introduced as a foundation for a causal explanation of the specific properties of material substances" (Bohr 1948, 313).

It is important to note that Bohr uses the term "individuality" in this passage (and in many similar ones) in a rather idiosyncratic way: although it does carry the standard meaning of referring to the individual, own nature of each single process or situation, the term equally refers to the indivisibility and the unanalyzable character of the whole (in accordance with the original meaning of "individuality"). This has not always been understood in the literature, with the result that some of Bohr's explanations have seemed mysterious.

Discreteness in interactions by itself obviously does not necessitate a renunciation of divisibility or analyzability. One can easily imagine a completely classical theory in terms of well-defined subsystems in which only discontinuous and finite energy exchanges occur, and even a classical theory in which such exchanges can be said to be "uncontrollable" is easy to conceive. To have an example of the latter, one might think of a scheme in which interactions follow purely probabilistic laws - this sacrifices the deterministic character of classical physics, but need not conflict with the classical principle that each of the parties in the interaction possesses its own independent state. In fact, a theory along precisely these lines was proposed by Bohr, 
Kramers and Slater in 1924. The experimental disproof of this "BKS theory" appears to have played a major role in Bohr's conclusion that a picture in which interacting quantum particles each possess their own description cannot be valid (Howard 2005). ${ }^{8}$

In the 1927 Como lecture Bohr states this conclusion right at the start as the very essence of the considerations that are to come: "Notwithstanding the difficulties which are involved in the formulation of the quantum theory, it seems, as we shall see, that its essence may be expressed in the so-called quantum postulate, which attributes to any atomic process an essential discontinuity, or rather individuality, completely foreign to the classical theories and symbolised by Planck's quantum of action" (Bohr 1928, 580). It is true that Bohr's formulation here (like in other places) is not as transparent as one would wish; but if one considers it in context and pays attention to Bohr's idiosyncratic use of "individuality", there is no doubt that Bohr is referring to the holistic nature of interacting systems. Any remaining hesitation on this point is removed when we read the further explanation that follows a few lines after the just-given quotation: "Now the quantum postulate implies that any observation of atomic phenomena will involve an interaction with the agency of observation not to be neglected. Accordingly, an independent reality in the ordinary physical sense can neither be ascribed to the phenomena nor to the agencies of observation" (Bohr 1928, 580) - italics added. ${ }^{9}$

In this quote the mention of an interaction that should not be neglected may seem to presuppose separate entities with well-defined states - states that are subsequently disturbed by the interaction. This is obviously not intended: the second sentence in the quote denies precisely this existence of independent states. Indeed, in several places in his writings Bohr warns us that when he uses phrases like "disturbing phenomena by observation", this should not be taken literally (Bohr 1948, 315; Bohr 1949, 237). So the "quantum postulate" should not be understood as the statement that energy

\footnotetext{
${ }^{8}$ Howard (2005) presents a detailed and illuminating survey of the physical developments that drove Bohr to his "wholeness" conclusion, and also discusses how Einstein dealt with these same developments. As Howard makes clear, it is in the early period of the history of quantum theory - 1905-1927 - that we can identify the roots both of Bohr's complementarity, and of Einstein's later resistance to quantum mechanics on the grounds that it does not satisfy a principle of separability. It is sometimes claimed that Bohr only came to embrace "holism" after the 1935 EPR discussion, but this is in conflict with the textual evidence. The doctrine already occurs in the 1927 Como lecture.

${ }^{9}$ By "agencies of observation" Bohr just means "measuring devices". Bohr never assigns a special role to human agency, apart from the capacity of human observers to decide to build one measuring apparatus rather than another.
} 
is exchanged in discrete packets, and that there is a random disturbance of prior existing properties by this exchange, but rather as a metaphorical expression of the fact that objects entering into an interaction come to form one whole ("as symbolised by the finite value of $h$ ", as Bohr often puts it).

Bohr's terminology certainly is apt to confuse here, because it makes use of expressions that already possessed a meaning but now acquire a new, metaphorical sense within the new conceptual framework. Bohr's global approach and way of arguing are very characteristic here: he comes to the subject as a physicist, adapting his intuitions on the basis of new empirical facts, and wrestles to make these new intuitions clear by couching them in familiar terms. This is completely different from the attitude of a philosopher starting from a desire to hygienically regiment language.

In Bohr's reply to EPR we again read:

Indeed the finite interaction between object and measuring agencies conditioned by the very existence of the quantum of action entails - because of the impossibility of controlling the reaction of the object on the measuring instruments if these are to serve their purpose - the necessity of a final renunciation of the classical ideal of causality and a radical revision of our attitude towards the problem of physical reality (Bohr 1935, 697).

No wonder that this passage has lead to misunderstandings, as it appears to deal explicitly with discrete interactions and disturbances. However, such a "common-sense reading" would make it completely mysterious why these finite interactions would "entail the necessity of a final renunciation of the classical ideal of causality and a radical revision towards the problem of physical reality" - a theme already familiar from the Como lecture. The mystery is solved when a little bit further on the same page we find: "The impossibility of a closer analysis of the reactions between the particle and the measuring instrument .... is an essential property of any arrangement suited to the study of the phenomena of the type concerned, where we have to do with a feature of individuality completely foreign to classical physics." This brings us back to wholeness and individuality (in the sense of indivisibility and "the impossibility of a closer analysis"), which is clearly what Bohr has in mind even when he uses expressions like "finite interactions between object and measuring device".

Note how easily Bohr switches, in the just-given pair of quotes, from "the impossibility of controlling the reaction of the object on the measuring instruments" to "the impossibility of a closer analysis of the reactions between the particle and the measuring instrument." The first expression, 
when taken literally, does not indicate at all that a drastic revision of classical concepts is necessary: the impossibility of controlling an interaction does not imply anything about the status of the various parties in the interaction. However, the impossibility of any further theoretical analysis may very well have such conceptual consequences. For Bohr, however, there seems to be no significant difference between the two expressions, which reinforces our earlier diagnosis: Bohr speaks as a physicist, using intuitive common sense language, without being too anxious about conceptual hairsplitting.

There can be no doubt that the metaphorical reading of "uncontrollable disturbance" is the correct one. It makes sense in all of Bohr's publications on the interpretation of quantum mechanics and, moreover, it plays a pivotal role in grounding the doctrine of "complementarity". For example, in the Einstein volume Bohr reviews once again experiments that illustrate the necessary "renunciation of the visualization of atomic phenomena imposed on us by the impossibility of their subdivision" (emphasis added), and draws the conclusion that "arguments of this kind which recall the impossibility of subdividing quantum phenomena ... reveal the ambiguity in ascribing customary physical attributes to atomic objects" (Bohr 1949, 222). He then goes on to point out that an unambiguous use of space-time concepts is only possible when classical concepts become applicable to one of the parties in the interaction, for example when marks have formed on a photographic plate in a practically irreversible amplification process. This then forges a connection to the doctrine of classical concepts and leads to the famous principle that definite properties can only be ascribed to atomic objects in a classically describable measurement context.

In his reply to EPR Bohr emphasizes that the ambiguity in ascribing physical properties to atomic systems is not a matter of insufficient knowledge. Rather, "we have not merely to do with an ignorance of the value of certain physical quantities, but with the impossibility of defining these quantities in an unambiguous way" (Bohr 1935, 699) - emphasis added. This lack of definiteness is in principle also there in the case of the description of the measuring device itself: it is subject to the uncertainty relations as well, as we shall further discuss below. But when macroscopic devices are at stake the smallness of the "latitudes" comes to the rescue (as we have seen in section 3): this makes them negligible and allows "a purely classical account of the measuring apparatus" (Bohr 1935, 698). So in the case of these measuring devices and other macroscopic objects it is consistent that we make epistemic contact with quantum reality by means of ordinary perception and classical concepts. Measuring devices can be described by means of values of classical quantities and by extension the measured atomic 
system, whose properties are correlated with the properties of the measuring device (pointer positions, dark spots on a photographic plate, and so on) becomes unambiguously describable as well.

We thus have returned to the theme of the indispensability of classical concepts of the previous section: measuring devices, like all macroscopic objects around us, can and must be described classically. It is an immediate consequence of this that measurements necessarily have only one single outcome. Pointers can only have one position at a time, a light flashes or does not flash, and so on - this is all inherent in the uniqueness of the classical description. Because of this, Bohr's interpretation does not face the "measurement problem" in the form in which it is often posed in the foundational literature, namely as the problem of how to explain - in the face of the presence of superpositions in the mathematical formalism - that there is only one outcome realized each time we run an experiment. For Bohr this is not something to be explained, but rather something that is given and has to be assumed to start with. It is a primitive datum, in the same sense that the applicability of classical language to our everyday world (see section 3 ) is a brute fact to which the interpretation of quantum mechanics necessarily has to conform. An interpretation that would predict that pointers can have more than one position, that a cat can be both dead and alive, etc., would be a non-starter from Bohr's point of view. So the measurement problem in its usual form does not exist; it is dissolved. This does not mean that we cannot or should not inquire how this "uniqueness of the classically describable world" fits in with the formalism of quantum theory, of course. We shall consider this question of the relation with the formalism in the next section.

For now, we want to return to another central point in Bohr's interpretation, namely the issue of how the classical description of the measuring device relates to complementarity, i.e. the doctrine that concepts like "position" and "momentum" cannot be applicable at the same time. If measuring devices were purely classical, so that the classical description applied in all exactness, there would be no reason to assume that there is an incompatibility between certain concepts: it is a hallmark of classicality that all physical quantities are well-defined and possess sharp values simultaneously (in mathematical terms: that the quantities form a Boolean algebra). If this were strictly true of the measuring device, there would be no problem of principle in attributing sharp values also to all quantities of correlated micro systems.

We have already seen in section 3 that Bohr denied that the classical descriptions on the macro level have a fundamental ontological import; macro 
systems are basically quantum mechanical. Still, in a famous part of his report about his discussions with Einstein, Bohr may appear to suggest that a completely classical account of measuring devices suffices to explain that it is impossible to assign both position and momentum to the measured quantum systems, as the experimental set-up needed to measure one of these quantities is incompatible with the set-up necessary to measure the other (Bohr 1949, 219-223). Thus, in order to measure position, one needs to fix all relative positions in the device by means of rigid connections, and the whole assembly should be bolted firmly to a common support. As Bohr comments, "in such an arrangement ... it is obviously impossible to control the momentum exchanged between the particle and the separate parts of the apparatus." By contrast, if we want to experimentally study momentum exchange, "certain parts of the whole device must naturally be given the freedom to move independently of others." These statements (famously illustrated by drawings that plastically exhibit the mutual exclusiveness of motion and stationarity) might create the impression that Bohr is contending that even on purely classical grounds one cannot simultaneously fix both momentum and position of a micro object. But this interpretation would be mistaken.

If the classical description applied with full precision, even the momentum absorbed by a device firmly bolted to the ground (in a laboratory on Earth) would be well-defined and could in principle be determined. Certainly, one would need a measuring procedure of fantastic accuracy for this purpose - but this is only a practical objection. By the same token, even the fastest moving classical object possesses at all times a well-defined position. Bohr is perfectly aware of this and leaves no doubt, in the passages in question, that it is only the basic quantum nature of the measuring devices that is responsible for complementarity.

For the case of a moving diaphragm with a slit in it, this means that "there will always be, in conformity with the indeterminacy principle, a reciprocal relationship between our knowledge of the position of the slit and the accuracy of the momentum control" (Bohr 1949, 220). ${ }^{10}$ Similarly, the impossibility of momentum control in the case of a stationary device is not a consequence of its classical stationarity, but is due to its quantum character. A very small latitude in position implies a very large quantum

\footnotetext{
${ }^{10}$ Bohr's use of the expressions "knowledge of the position of the slit" and "accuracy of the momentum control" may suggest that there are in fact precise positions and momenta, although we do not know them; but this is not what he intends, as we have seen before and as he explains on p. 237 of the same text. It is the unsharpness of definition and the corresponding "latitude" that are decisive here.
} 
latitude (indeterminacy, "uncertainty") in momentum and it is only this that makes it impossible to control the exchanged momentum. The bolting to the ground (which is equivalent to giving the device a huge mass) only serves to guarantee that the latitude in velocity associated with the large latitude in momentum can be completely neglected, so that we have a truly stationary device, as described in classical terms.

In other words, the doctrine of complementarity in its relation to measurement contexts only makes sense if it is acknowledged that measuring devices, like macroscopic objects in general, are in principle subject to quantum mechanics, even if it is true that we need classical concepts to have epistemological access to them. This reiterates our earlier point that the use of common language, extended by classical physics, is an epistemological manoeuvre that does not imply any ontic divide.

That the structure of the quantum formalism, in spite of its "symbolic character", was a decisive factor in the formation of Bohr's thoughts about complementarity is something we have already seen in section 2 . In the Como lecture Bohr used the superposition principle and de Broglie wave packets to argue for the uncertainty relations, conceived as limitations on sharp definition, and based complementarity on this. In his reply to Einstein, Podolsky and Rosen, in the Einstein volume, and in his Dialectica article Bohr explicitly applies the uncertainty relations to macroscopic objects in order to justify complementarity. As he puts it in the EPR paper:

in the phenomena concerned we are not dealing with an incomplete description characterized by the arbitrary picking out of different elements of physical reality at the cost of sacrificing other such elements, but with a rational discrimination between essentially different experimental arrangements and procedures which are suited either for an unambiguous use of the idea of space location, or for a legitimate application of the conservation theorem of momentum. ... In fact, the renunciation in each experimental arrangement of the one or the other of two aspects of the description of physical phenomena,- - the combination of which characterizes the method of classical physics, and which therefore in this sense may be considered as complementary to each other,- - depends essentially on the impossibility, in the field of quantum theory, of accurately controlling the reaction of the object on the measuring instruments, i.e., the transfer of momentum in the case of position measurements, and the displacement in case of momentum measurements. Just in this 
last respect any comparison between quantum mechanics and statistical mechanics,- - however useful it may be for the formal representation of the theory, - is essentially irrelevant. Indeed we have in each experimental arrangement suited for the study of proper quantum phenomena not merely to do with an ignorance of the value of certain physical quantities, but with the impossibility of defining these quantities in an unambiguous way (Bohr 1935, 699).

This may not be the most transparent and elegant way to explain the situation, but still, all the important elements are here. Whether a given set-up is appropriate for fixing position or momentum of a micro object is not a choice up to us, but is decided by whether the instantiated quantum properties are either close enough to classical position or to momentum. According to quantum theory, these two possibilities are mutually exclusive. This is not due to some epistemic conundrum, but mirrors a feature of quantum reality: position and momentum cannot be sharply defined at the same time, not even for macro objects.

As we have seen in the beginning of this section, the "uncontrollable disturbance" and "individuality" that Bohr frequently mentions as being a necessary part of any interaction, in particular a measurement, are meant to refer to the holistic nature of the combined interacting system. Accordingly, Bohr says, in a measurement the "micro part" does not possess its own independent state. So the situation is not like the one in classical mechanics in which a particle can always - whether in a measurement situation or notbe characterized by its position and momentum values. This means that we can only attribute position or momentum to the micro system relative to the actual measurement context in which it finds itself. This then is the essential ingredient of the complementarity doctrine: a micro system, for example an electron, can only be assigned a position or a momentum relative to a concrete context.

Given that even macroscopic objects - like measuring devices - are basically quantum mechanical, consistency requires that their properties should in principle be contextual as well. That implies that also their properties depend on the measurement context. Bohr does not discuss this in depth: he only remarks, as we have seen, that if objects are sufficiently big and heavy quantum effects can be neglected for all practical purposes; and that we should only consider the remaining very small latitudes in their classical properties if consistency requirements force us to treat macroscopic objects as explicitly quantum mechanical. As we shall see, more can be said about 
this and similar issues if we take account of the quantum formalism in a more detailed way than we have done, in the footsteps of Bohr, so far.

\section{Bohr's interpretation and the formalism}

We can conclude from our discussion up to now that the formal structure of quantum mechanics played a role in Bohr's interpretation that is more important than standard stories tell us. True, Bohr saw the formalism as "symbolic"; by this he meant that a visualizable classical interpretation was not possible. But he did not at all think that the formalism should be considered a mathematical calculus without physical content. Quite the opposite: the structure of the formalism gave Bohr the essential motivation for his doctrine of complementarity, via the uncertainty relations and the canonical commutation relations. Complementarity entails an account of what the physical world is like: it implies a "relativization" of the applicability of classical concepts to the quantum world and thus tells us how the physical structure of the micro world should be characterized. Moreover, the interpretation of the mathematically defined uncertainties as "indeterminacies in definition", "latitudes", in classical quantities made it possible for Bohr to combine the epistemologically unavoidable classical descriptions of macroscopic objects in the everyday world around us with their inherent quantum character.

It remains true that Bohr appealed only to general features of the quantum formalism: all he used was some Fourier analysis of wave packets, the operator form of $p$ and $q$ and the canonical commutation relations, the superposition principle for waves, and a number of qualitative statements about the role of macroscopic masses and irreversibility in going to the classical limit. In addition, Bohr commented on the importance of the fact that wave functions of many-particles systems are defined in high-dimensional $n$-particles configuration spaces, and that these wave functions cannot be reduced to $n$ three-dimensional waves. As we have seen, the latter is due to the validity of the superposition principle in the configuration space, which in general makes the total wave function non-factorizable. In present-day parlance, the $n$-particles wave function generally is an entangled state. It is now recognized that entanglement is one of the most characteristic features of quantum mechanics - if not the most characteristic feature, as famously suggested by Schrödinger (1935). Although Bohr in effect referred to entanglement as his most important ground for thinking that the formalism has a non-pictorial content, he did not go into further details and did not explic- 
itly single out entanglement (or an equivalent notion) as a key mathematical feature of the formalism.

He did, however, single out the "individuality" and wholeness occurring in quantum interaction processes as an essential and revolutionary trait of the new theory. From a modern point of view this wholeness, according to which systems do not keep their own wave functions during an interaction, is a direct consequence of entanglement. When two systems - each in their own quantum state - start to interact, the Schrödinger equation (more generally: any unitary evolution equation) dictates that an entangled twoparticles state is formed in which the component systems can only be described by "mixtures" (obtained by "partial tracing") instead of pure states (wave functions). So one is tempted to relate Bohr's emphasis on the vital importance of "individuality" in the interpretation of quantum mechanics to the pivotal role of entanglement in modern more formal considerations. In particular, when Bohr speaks about the inextricable whole formed by measuring device and quantum system (vital for complementarity), this seems translatable into statements about the combined entangled state of device and object system that results from the interaction between them. ${ }^{11}$ So one could argue, as Howard suggests, that Bohr was familiar with entanglement and recognized its importance in the formalism (Howard 1994, 2004, 2005).

There can be no doubt that Bohr indeed knew about consequences of entanglement, for example from his discussion with Einstein, Podolsky and Rosen, and had even seen its formal representation in von Neumann's theory of measurement (von Neumann 1932). Still, it seems less than certain that he actually thought about his "individuality" along lines suggested by the formal treatment of entangled states à la von Neumann. Bohr's way

\footnotetext{
${ }^{11}$ This assumes that Bohr did not suppose that there exists a special form of interaction, resulting in a "collapse of the wave function", manifesting itself only in measurements (as opposed to ordinary physical interactions). This non-collapse assumption is eminently plausible, given that Bohr always speaks about interactions between measuring devices and objects in ordinary physical terms, such as "uncontrollable disturbance", "discrete exchange of energy and momentum", leading to the typical "individuality of the phenomenon". Moreover, as we have seen, Bohr in many places stresses the universality of quantum theory, which is hardly compatible with a special role for macroscopic measuring instruments as far as physical evolution is concerned. Finally, Bohr never mentions "collapses" in his published writings. Nevertheless, it has been part of the folklore surrounding the "Copenhagen interpretation" to maintain that Bohr's views are characterized precisely by such "collapses" - this is now generally rejected by Bohr scholars (Howard 2004; Faye 2014). Zinkernagel (2016) has recently drawn attention to a number of unpublished letters in which Bohr mentions the updating of our knowledge when measurement results become available, which seems similar to collapses. But this updating of information can easily be given an epistemic interpretation.
} 
of speaking about "individuality" and wholeness always kept a flavor of the old quantum theory, appealing as it did to an interaction that cannot be analyzed and therefore blurs the distinction between the partners in the interaction. It is true that Bohr came to use this terminology in a metaphorical way, but he never made a transition to an explicit discussion of formal aspects of entanglement in this context. We must of course realize that nobody did so at the time, so the question of whether Bohr was really aware of the significance of entanglement may be too anachronistic to deserve a debate.

However this may be, I think it is safe to say that Bohr's "individuality" actually corresponds to entanglement in the formalism, and that by using this we may translate - or "reconstruct", as Howard (1994) says-Bohr's statements in formal terms, regardless of whether Bohr himself was thinking in terms of this precise formal correspondence.

With the help of this translation of "individuality" into "entanglement" a formal picture of Bohr's account of quantum mechanics can be constructed. To start, let us translate into mathematical terms Bohr's claim that two interacting quantum systems generally lose their own states and become part of one "individual" whole. In the Hilbert space formalism this is appropriately captured by the standard von Neumann form of a unitary interaction:

$$
\left|S_{0}^{1}\right\rangle \otimes\left|S_{0}^{2}\right\rangle \longrightarrow \sum_{i} c_{i}\left|S_{i}^{1}\right\rangle \otimes\left|S_{i}^{2}\right\rangle,
$$

in which $\left|S_{0}^{1}\right\rangle$ and $\left|S_{0}^{2}\right\rangle$ are the initial states of system 1 and system 2, respectively, and in which the arrow represents the unitary evolution that results from the interaction. The right-hand side of Eq. (1) is an entangled state, which for convenience has here been written in its Schmidt form (so that the states $\left|S_{i}^{1}\right\rangle$, and also $\left|S_{i}^{2}\right\rangle$, are mutually orthogonal for values of $i$ that differ from each other). In this entangled state no single (pure) state can be assigned to either system 1 or system 2 ; the best one can do is to extract, by "partial tracing", a density operator for each of the two systems: $\sum_{i}\left|c_{i}\right|^{2}\left|S_{i}\right\rangle\left\langle S_{i}\right|$ (for ease of notation the index 1 or 2 has been suppressed).

This then is the mathematical Hilbert space translation of Bohr's statement that the separate systems do not possess their own wave functions when interactions are going on; and of the claim that the whole system displays an "individuality" typical of quantum mechanics - "individuality" being represented by entanglement.

The interesting special case of course is the one in which we are dealing with a measurement, in the sense of an interaction between a quantum system and a macroscopic system in which an irreversible recording of an 
outcome takes place. As we have seen, this is the situation used by Bohr to inject physical content into the formalism: macroscopic devices can be characterized by sharp values of position, momentum and other classical quantities and this characterization gives us epistemic access to the quantum world. In order to formalize this thought, we have to look for representations of interactions in which at least one of the systems can be described classically; that is, it should be represented in the formalism with quantum "latitudes" that are so small that the attribution of sharp classical values becomes possible without practical contradictions.

Bohr himself gives us the hint that we should think of big and heavy systems that are able to amplify micro differences in an irreversible way, so that well-defined measurement results arise. This accords well with he present-day consensus that decoherence processes are vital for understanding the classical limit of quantum mechanics. The core principle of decoherence is that systems which are sufficiently big will in ordinary circumstances be in continuous interaction with their environment, and that this interaction will wash out the quantum aspects of the behavior of the system. Typical interactions in our macroscopic environment (primarily electromagnetic interactions) are sensitive to the positions of the interacting objects and this has the consequence that macro objects, through the irreversible "decohering" interaction with their environment, will lose quantum coherence between different positions - which in turn means that they will behave as if they possessed a definite position at each moment. More precisely, measurement interactions involving classically describable devices have a form like:

$$
\left|S_{0}\right\rangle \otimes\left|M_{0}\right\rangle \otimes\left|E_{0}\right\rangle \longrightarrow \sum_{i} c_{i}\left|S_{i}\right\rangle \otimes\left|M_{i}\right\rangle \otimes\left|E_{i}\right\rangle,
$$

where $S$ denotes the micro system on which a measurement is being made, $M$ is the macroscopic measuring device, and $E$ stands for the decohering environment that is in continual interaction with the device. The idea is that because of the interaction with the environment, the $\left|M_{i}\right\rangle$ Hilbert space states will be (practically) mutually orthogonal and entangled with orthogonal environment states $\left|E_{i}\right\rangle$ - this is the decoherence effect that washes out interference between different values of $i$. Furthermore, the states $\left|M_{i}\right\rangle$ will be characterized by macroscopic pointer position values with a very small indeterminacy (i.e., $\left\langle M_{i}\left|(X-\bar{X})^{2}\right| M_{i}\right\rangle$ vanishingly small in comparison to $\bar{X}$, with $X$ the relevant position operator and $\bar{X}$ its expectation value). The irreversible interaction between the measuring device and the environment $E$ keeps these practically classical position values intact, in the sense that 
sharp values will not be blurred over time. Finally, the interaction between $S$ and $M$ should represent a measurement of some observable, for example momentum $p$. That means that different positions of the almost-classical pointer (on the dial of the device) should become correlated, by the interaction, to different momentum states of the system $S$ (so that the Hilbert space states $\left|S_{i}\right\rangle$ of Eq. (2) in this case should be momentum eigenstates).

This account is only a rough sketch of how macroscopic measurements are modeled, with the help of decoherence, in present-day quantum mechanics; Rosaler (2016) gives a more detailed account. The essential point is that classical structures can be identified in the formal quantum representation, in the sense that device states with almost classical properties naturally occur (i.e. states with practically sharp values of position together with practically sharp values of velocity, obeying classical equations of motion with high precision). This then is the point where the formalism allows contact with Bohr's epistemological demand that we must describe the world around us in classical terms. So the (almost-classical) state $\left|M_{i}\right\rangle$ of the measuring device represents the situation that we describe in classical terms with a pointer position " $i$ ", and we assign the correlated value of the measured quantity to the micro system $S$. On the micro level the indeterminacies (latitudes) can of course not be neglected, and the quantum formalism automatically fulfils the requirements of complementarity in the form of the uncertainty relations for canonically conjugated quantities. So quantities like position and momentum can never be assigned sharp values together. The nature of the measuring device and its interaction with the system, finally resulting in a state of the form of the right-hand side of Eq. 2, fixes which properties can be assigned to the object (namely those that are definite-valued in the states $\left.\left|S_{i}\right\rangle\right)$.

We have glossed over an important point however. In Eq. 2, as in all equations representing final states after unitary interactions, we do not have one state of the measuring device, but all these states $\left|M_{i}\right\rangle$ figure together in the superposition at the right-hand side of the arrow. So in the Bohrian interpretation of the formalism that we just explained we seem to have committed the error of replacing the full superposition by just one term in it. In fact, this is the standard objection against claims that decoherence solves the measurement problem. In the quantum formalism with only unitary evolution (so no collapses) we always end up with superpositions after interactions, also in the case of decoherence (as can be verified by looking at Eq. 2 ). But this means, so the objection goes, that the measurement problem, in the form of the question why there is only one outcome, has not been solved - and even cannot be solved. 
However, this objection misconstrues Bohr's approach to the interpretational problem, as we have already explained in section 4. Although Bohr takes the mathematical formalism of quantum mechanics seriously, as providing us with information about the micro world, he does not start from the formalism in his interpretation. The formalism can only tell us about properties of micro systems once it has made contact with physical reality, and this contact can only be established via identifications between symbols and physical quantities on the macro level, described in classical language. The starting point for attributing physical meaning is thus the classical description of laboratory practice - and here experiments certainly have only one result at a time. So the uniqueness of experimental outcomes is not something to be explained, but is something accepted from the outset. The task is to find suitable expressions in the formalism that can represent these unique outcomes, and in this way to endow the mathematics with physical content.

In other words, the mistake made in the objection is the supposition that the mathematical framework, with its superpositions, already possesses physical meaning before the empirical interpretation has even started, and that the occurrence of superpositions of states $\left|M_{i}\right\rangle$ must signal the joint physical presence of all results $i$. In the Bohrian approach that we are outlining this is putting the cart before the horse. The measurement problem in its usual form, coming from the urge to explain how superpositions can fail to correspond to multiple outcomes, does not exist for Bohr.

In order to make this Bohrian approach correspond to the mathematical formalism, we need the following interpretational move: in measurement situations in which the final formal state is of the form $\sum_{i} c_{i}\left|S_{i}\right\rangle \otimes\left|M_{i}\right\rangle \otimes\left|E_{i}\right\rangle$, as in Eq. 2, the corresponding physical situation is that the pointer of the device points to one of the possible pointer positions $i$; the probability that a specific $i$ instead of one of the alternative values is realized is given by $\left|c_{i}\right|^{2}$.

This interpretational manoeuvre is not only Bohrian, but in accordance with a wide class of non-collapse interpretations of quantum mechanics. In particular, modal interpretations employ an explicit interpretational rule of precisely this kind (Lombardi and Dieks 2014; Bub 1997; Dieks 1989a,b); but the many-histories interpretation and the Everett interpretation are also compatible with the idea that our observable world corresponds to one branch from the superposition, selected by decoherence (Rosaler 2016). We shall briefly return to this connection with non-collapse interpretations in the next section.

The proposal to interpret the superposition in terms of one single out- 
come bears similarity to Howard's "reconstruction" of Bohr (Howard 1994). Howard reads Bohr as saying that the description of the measuring device by the superposition of Eq. 2 can be replaced by one of the components in the density operator of $M$. According to Howard, Bohr proposes that we act "as if" the right-hand side of (2) were $\left|S_{i}\right\rangle \otimes\left|M_{i}\right\rangle \otimes\left|E_{i}\right\rangle$. But it is misleading to introduce such an "as if". It suggests that the full superposition by itself cannot have the desired meaning, and that Bohr therefore had to have recourse to a sleight of hand. But as we have just argued, the superposition does not have an empirical meaning independently of its interpretation via classically described experiments, so no replacement by another mathematical state is needed. We just have to interpret the formulas correctly.

One might get the impression that the interpretation of entangled states of object system plus a measuring device in terms of just one measurement result is a verbal move that is equivalent to accepting the projection postulate. If this were right, the interpretation would bring in the projection postulate via the back door, and would effectively violate what we have accepted as one of Bohr's principles, namely that projection or collapse should not be seen as a dynamical evolution process, but rather as a way of efficient bookkeeping that takes into account available information about experimental outcomes. However, there is an important difference between the interpretational principle outlined above and the projection postulate. The projection postulate tells us that the quantum mechanical state, as defined in the mathematical formalism, changes in a measurement from an entangled state to a product state, namely one of the terms occurring in the original entangled superposition. This is a non-unitary evolution that is impossible in non-collapse interpretations. In principle, this difference in the sorts of interaction that occur in the formalism can lead to a difference in empirical predictions. The reason is that if we do not have collapses, superpositions will always maintain themselves, and this entails the possibility in principle of interference between the "branches" defined by the individual product states; whereas in the case of collapses no other branches are left with which interference can take place. It is true that this possibility of interference is remote in the circumstances of ordinary experiments, and Bohr probably never considered such exotic consequences of quantum theory. Moreover, his emphasis on the irreversibility of measurements, which is vindicated by the modern decoherence program, effectively blocks the possibility of a recombination of branches in ordinary experimental practice. Nevertheless, experimental techniques have advanced enormously since Bohr's days, and it is not excluded that effects of unitarity will become accessible to empirical tests. 
Given that modern experimental techniques have created possibilities that were unheard of in Bohr's days, there may also be ways to investigate other (though related) possible effects of unitarity on the macroscopic level. Think of a hermetically closed chamber, in which a quantum measurement takes place. According to the interpretation of entangled states that we have outlined, a macroscopic device inside the chamber will register one unique result, even though according to the unitary theory the state of quantum system plus device is a superposition. However, an external observer who performs measurements on this room from the outside may be able to find results that indicate that the formal description of the chamber with its contents by the superposition is the right one (Bene and Dieks 2002; Dieks 2016).

The scenario reminds one of the predicament of Schrödinger's famous cat, which is shut up in a sealed room together with a decaying atom that is able to activate an infernal machine. After some time, the macroscopic cat system plus its microscopic environment will be formally described by an entangled superposed state. According to the Bohrian interpretation of the formalism as we have discussed it here, the cat is either dead or alive after the experiment. However, an external observer who performs measurements on the closed room, and thus has no direct access to the cat, can in principle find out that the superposed formal "dead plus alive state" is correct. For example, this superposed state may be an eigenstate of the observable measured by the external observer, and this can be borne out by the results of experiments. Experiments with such "Schrödingercat states" have actually been performed during the last two decades. The results show that an external observer can indeed observe the existence of the superposition, and thus verify that no collapse has taken place.

We thus see that the relational character of Bohr's interpretation must even go further than he himself emphasized, if we take its strict relation to the mathematical formalism seriously. Bohr stressed that the physical state of a system (in terms of the physical properties that can be attributed to it) only becomes definite in a well-defined experimental context. Thus, an electron can be assigned a position in the context of a position measurement, or a momentum value in the context of a measurement of momentum; and these two property assignments never apply simultaneously. But now an additional relational aspect reveals itself: for an observer inside the room with the cat (in the Schrödinger experiment) the cat is either dead or alive, but for an outside observer this need not be the case. So properties not only need "defining circumstances" to make them definite, but can also vary from one observer to another in (admittedly far from common!) circumstances of 
the kind we just described (Bene and Dieks 2002; Dieks 2016).

Even in such very strange situations, classical terminology remains applicable, in accordance with Bohr's claim. Thus, the internal observer describes the cat as either dead or alive, and the external observer establishes that the pointer of his measuring instrument, with which he performs his measurement on the sealed room, adopts a definite position that indicates the result. But the prediction and explanation of these measurement results cannot be given along classical lines; it needs the full (non-collapse) formalism of quantum mechanics. This illustrates Bohr's earlier-quoted dictum that "however far the phenomena transcend the scope of classical physical explanation, the account of all evidence must be expressed in classical terms" (Bohr 1949, 209).

\section{Conclusion}

Although Bohr qualified the mathematical formalism of quantum theory as symbolic, he took it more seriously than usually acknowledged. He realized that physical meaning has to be injected into the formalism in order to make it a physical theory, and took the pragmatically and epistemologically well-justified position that this can only be done via contact with our world of experience, which we describe in common language enriched with terms from classical physics. However, the form of the formalism itself stands in the way of implementing a full classical interpretation across the board, extending to the micro domain. Classical pictures of micro systems are therefore impossible, according to Bohr, and this is responsible for the fact that the formalism is symbolic even after empirical interpretation, namely in the sense that it does not support a vizualizable representation of the micro world - at least not in the sense of the pictures we normally associate with physical systems. This does not mean that according to Bohr we should opt for instrumentalism and forswear talk about electrons, photons and atoms. Such quantum systems do exist, but their mode of being cannot be captured by describing them with independent properties and states. This is all made more precise by the doctrine of complementarity, as we have seen in sections 2, 3 and 4. Significantly, structural features of the quantum formalism (superposition of waves, the canonical commutation relations) inspired Bohr decisively in this doctrine.

There can be little doubt that Bohr saw quantum mechanics as a universal theory, with an unrestricted domain of validity. It is also highly plausible that he did not think of the collapse of the wave function as a physical pro- 
cess, as a rival in the formalism of unitary Schödinger time evolution. From a modern perspective, this places Bohr in the camp of non-collapse interpretations of quantum mechanics (section 5).

The latter observation raises the (partly historical) question of why Bohr did not embrace attempts to formulate formally precise versions of noncollapse interpretations, in particular why he remained hostile to the "relative state" interpretation proposed by Everett in his dissertation (Everett 1957). Significantly, Everett's supervisor Wheeler was convinced that Everett's work could be seen as a formal elaboration of Bohr's interpretation and started a correspondence about the subject with the Copenhagen group; Everett even visited Bohr on Wheeler's instigation in 1959 (after two earlier aborted plans to visit). But Everett's work was not received favorably. ${ }^{12}$

From the evidence adduced by Osnaghi, Freitas and Freire (2009) it seems clear that Bohr's main objection was that it remained completely unsolved how Everett's scheme made contact with physical reality. Everett's universal wave function struck the Copenhagenists as a purely formal piece of mathematics, because it did not explicitly relate to an experimental context which can be described in classical terms. So the spot where the shoe pinched was in the epistemological part of Bohr's interpretation, the doctrine of the inevitability of the use of classical concepts (section 3). Indeed, both Everett and Wheeler relativized the inevitability of classical concepts and thought of the quantum formalism as a more general picture of quantum reality (although Wheeler later retraced his steps). This appears to highlight a significant difference between Everett and Bohr: although Bohr was not an instrumentalist, his views were firmly bound up with the experimental practice of physics, and he took the concepts that derive from this practice as primordial. Accordingly, any consideration of the formalism and its meaning should be preceded by the specification of physical concepts that ultimately derive from the description of macroscopic experimental situations. This need not be regarded as a Kantian move: it is first and foremost

\footnotetext{
${ }^{12}$ This historical episode is described in an informative article by Osnaghi, Freitas and Freire (2009). This article contains a detailed account of the correspondence between Wheeler and various members of the Copenhagen group, which makes it clear that Bohr himself did not seriously study Everett's work and left it to his collaborators to respond. They did so in ways that were not always consistent with each other, nor with Bohr's own general views. Part of Bohr's lack of interest may have been due to Everett's unforthcoming attitude with respect to "Copenhagen" - which was repaid in kind. However this may be, a basic theme can be distilled from the few remarks by Bohr himself that have been passed down and from the diverse arguments coming from Bohr's collaborators. This theme is well described by Osnaghi, Freitas and Freire (2009), even though these authors place their findings against the mistaken backdrop of Bohr's supposed instrumentalism.
} 
a pragmatic physicist's attitude that serves to safeguard the direct link to concrete physical experience. By contrast, Everett's attitude was completely abstract. Everett did not delve into the epistemological problem of how the mathematical symbols receive their physical meaning and did not analyze physical practice. It is no wonder then that the Copenhagen camp considered Everett as someone who had dabbled at an abstract mathematical structure and naively took it for a picture of the world, without asking himself what the picture-reality relation consisted in. For them, he was someone who had not even understood what the interpretational question was about.

This intermezzo further clarifies Bohr's attitude with respect to the quantum formalism. He certainly took the formalism seriously, was not an instrumentalist and was guided by the mathematical structure of the formalism when he developed his interpretation. But physical concepts, which he took to be indispensable for providing meaning to the formalism, always remained the most important ingredient in his analyses. It remains to be seen, however, whether from a present-day point of view Bohr's objections to Everett provide weighty arguments against an interpretation of Bohr's interpretation as a non-collapse interpretation in the modern sense. As far as I can see, Bohr's doctrine of classical concepts may very well be incorporated in formal non-collapse schemes, even if these make use of the notion of a universal wave function. This, however, is better kept for another study.

\section{References}

Beller, M. 1999. Quantum Dialogue: The Making of a Revolution. Chicago: University of Chicago Press.

Bene, G., and D. Dieks. 2002. "A Perspectival Version of the Modal Interpretation of Quantum Mechanics and the Origin of Macroscopic Behavior." Foundations of Physics 32: 645-71.

Bohr, N. 1928. "The Quantum Postulate and the Recent Development of Atomic Theory." Nature 121: 580-90. Reprinted in (Bohr 1985, 109-36).

Bohr, N. 1935. "Can Quantum-Mechanical Description of Physical Reality Be Considered Complete?" Physical Review 48: 696-702.

Bohr, N. 1948. "On the Notions of Causality and Complementarity." Dialectica 2: 312-19. 
Bohr, N. 1949. "Discussions with Einstein on Epistemological Problems in Atomic Physics." In Albert Einstein: Philosopher-Scientist, edited by P. A. Schilpp, 201-41. La Salle: Open Court. Reprinted in (Bohr 1985, 9-49).

Bohr, N. 1985. Collected works, Vol. 6: Foundations of Quantum Mechanics I (1926-1932). Edited by J. Kalckar. Amsterdam: North-Holland.

Bub, J. 1997. Interpreting the Quantum World. Cambridge: Cambridge University Press.

Camilleri, K., and M. Schlosshauer. 2015. "Niels Bohr as Philosopher of Experiment: Does Decoherence Theory Challenge Bohr's Doctrine of Classical Concepts?" Studies in History and Philosophy of Modern Physics 49: $73-83$.

Cushing, J.T. 1994. Quantum Mechanics: Historical Contingency and the Copenhagen Hegemony. Chicago: University of Chicago Press.

Dieks, D. 1989. "Quantum Mechanics Without the Projection Postulate and its Realistic Interpretation." Foundations of Physics 19: 1395-1423.

Dieks, D. 1989. "Resolution of the Measurement Problem Through Decoherence of the Quantum State." Physics Letters A 142: 439-46.

Dieks, D. 2016. "Information and the Quantum World." Entropy 18: 26. doi:10.3390/e18010026.

Drieschner, M., editor. 2014. Carl Friedrich von Weizsäcker: Major Texts in Physics. Heidelberg: Springer.

A video recording of Teller recounting the story himself can be found on the website http://www.webofstories.com/play/edward.teller/32 (visited January 2016).

Everett, H. 1957. "Relative State' Formulation of Quantum Mechanics." Reviews of Modern Physics 29: 454-62.

Faye, J. 2008. "Niels Bohr and the Vienna Circle." In The Vienna Circle in the Nordic Countries, edited by J. Mannheim and F. Stadler, 4-10. Berlin: Springer.

Faye, J. 2014. "Copenhagen Interpretation of Quantum Mechanics." In The Stanford Encyclopedia of Philosophy (Summer 2014 Edition), edited by E.N. Zalta. Stanford: Metaphysics Research Lab of Stanford University. 
Folse, H. 1985. The Philosophy of Niels Bohr: The Framework of Complementarity. Amsterdam: North-Holland.

Howard, D. 1994. "What Makes a Classical Concept Classical? Toward a Reconstruction of Niels Bohr's Philosophy of Physics." In Niels Bohr and Contemporary Philosophy, edited by J. Faye and H. Folse, 201-29. Dordrecht: Kluwer.

Howard, D. 2004. "Who Invented the "Copenhagen Interpretation? A Study in Mythology." Philosophy of Science 71: 669-82.

Howard, D. 2005. "Revisiting the Einstein-Bohr Dialogue." Available at: https://www3.nd.edu/ dhoward1/Revisiting

Landsman, N.P. 2006. "When Champions Meet: Rethinking the BohrEinstein Debate." Studies in History and Philosophy of Modern Physics 37: $212-42$.

Landsman, N.P. 2007. "Between Classical and Quantum." In Handbook of the Philosophy of Science, Vol. 2: Philosophy of Physics, edited by J. Earman and J. Butterfield, 417-554. Amsterdam: Elsevier.

Lombardi, O., and D. Dieks. 2014. "Modal Interpretations of Quantum Mechanics." In The Stanford Encyclopedia of Philosophy, Spring 2014 Edition, edited by E.N. Zalta. Stanford: Metaphysics Research Lab of Stanford University.

Osnaghi, S., F. Freitas, and O. Freire Jr. 2009. "The Origin of the Everettian Heresy." Studies in History and Philosophy of Modern Physics 40: 97-123.

Rosaler, J. 2016. "Interpretation Neutrality in the Classical Domain of Quantum Theory." Studies in History and Philosophy of Modern Physics 53: $54-72$.

Schrödinger, E. 1935. "Die gegenwärtige Situation in der Quantenmechanik." Die Naturwissenschaften 23: 807-12.

Von Neumann, J. 1932. Mathematische Grundlagen der Quantenmechanik. Berlin: Springer.

Zinkernagel, H. 2015. "Are we Living in a Quantum World? Bohr and Quantum Fundamentalism." In One hundred years of the Bohr atom: Proceedings from a conference. Scientia Danica, Series M: Mathematica et physica, vol. 1, edited by F. Aaserud and H. Kragh, 419-34. Copenhagen: Royal Danish Academy of Sciences and Letters. 
Zinkernagel, H. 2016. "Niels Bohr on the Wave Function and the Classical/Quantum Divide." Studies in History and Philosophy of Modern Physics 53: 9-19. 